International Journal of Pure and Applied Mathematics

Volume 110 No. 1 2016, 9-26

ISSN: 1311-8080 (printed version); ISSN: 1314-3395 (on-line version)

url: http://www.ijpam.eu

doi: 10.12732 /ijpam.v110i1.2

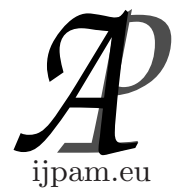

\title{
OSCILLATION AND ASYMPTOTIC BEHAVIOR OF NEUTRAL ADVANCED DIFFERENCE EQUATIONS
}

\author{
A. Murugesan ${ }^{1 \S}$, K. Shanmugavalli ${ }^{2}$ \\ ${ }^{1}$ Department of Mathematics \\ Government Arts College (Autonomous) \\ Salem, 636 007, Tamil Nadu, INDIA \\ ${ }^{2}$ Department of Mathematics \\ Government Arts College for Women \\ Salem, 636 008, Tamil Nadu, INDIA
}

Abstract: In this paper, we discuss the oscillation and asymptotic behavior of the first order neutral difference equation of the form

$$
\Delta[x(n)+p x(n+\tau)]-\sum_{i=1}^{r} q_{i}(n) f\left(x\left(n+\sigma_{i}\right)\right)=0, \quad n \geq n_{0},
$$

where $p$ is a real number, $\tau, \sigma_{1}, \sigma_{2}, \ldots, \sigma_{r}$ and $r$ are positive integers, $\left\{q_{i}(n)\right\}, i=1,2, \ldots, r$ are sequences of nonnegative real numbers and $f: R \rightarrow R$ is a real valued function.

AMS Subject Classification: 39A10, 39A12

Key Words: neutral delay difference equations, advanced difference equation, oscillation and asymptotic behavior

\section{Introduction}

Recently, there has been a great deal work on the oscillatory and asymptotic behavior of solutions of first order neutral delay difference equations. In particular, several researchers discussed the problem of finding sufficient conditions

Received: $\quad$ March 17, 2016

Revised: $\quad$ April 28, 2016

Published: October 21, 2016

(c) 2016 Academic Publications, Ltd.

url: www.acadpubl.eu

${ }^{\S}$ Correspondence author 
for oscillation of all solutions and for all solutions to tend to zero, see, for example, the papers [4], [8]-[10]. On the other hand, considerably less is known about the asymptotic and oscillatory solutions of first order neutral advanced difference equations. We choose to refer to the papers [3], [7] and also to the monographs [1], [2], [5].

In this paper, we consider the first order neutral advanced difference equation

$$
\Delta[x(n)+p x(n+\tau)]-\sum_{i=1}^{r} q_{i}(n) f\left(x\left(n+\sigma_{i}\right)\right)=0, \quad n \geq n_{0},
$$

where $\Delta$ is the forward difference operator defined by $\Delta x(n)=x(n+1)-x(n)$, $p$ is a real number, $r$ is a positive integer, $\sigma_{1}, \sigma_{2}, \ldots, \sigma_{r}$ and $\tau$ are positive integers, and $\left\{q_{i}(n)\right\}, i=1,2, \ldots, r$ are sequences of nonnegative real numbers which are not identically zero, for large values of $n$. Also $f: R \rightarrow R$ is a real valued function such that $u f(u)>0$ for $u \neq 0$.

By a solution of (1) on $N\left(n_{0}\right)=\left\{n_{0}, n_{0}+1, n_{0}+2, \ldots\right\}$, we mean a real sequence $\{x(n)\}$ which is defined on $N\left(n_{0}\right)$ and which satisfies (1) for $n \in$ $N\left(n_{0}\right)$. A solution $\{x(n)\}$ of $(1)$ on $N\left(n_{0}\right)$ is said to be oscillatory if for every positive integer $N_{0}>n_{0}$ there exists $n \geq N_{0}$ such that $x(n) x(n+1) \leq 0$, Otherwise $\{x(n)\}$ is said to be non-oscillatory. The difference equation(1) is called oscillatory if all its solutions are oscillatory. Otherwise, it is called nonoscillatory.

Huong [6] discussed the oscillatory and asymptotic behavior of the equation (1) for the case $\tau$ and $\sigma_{i}(i=1,2, \ldots r)$ are negative integers.

In this paper, our aim is to establish sufficient conditions for oscillation of all solutions of (1) and for asymptotic behavior of solutions of (1).

\section{Main Results}

\subsection{The Oscillation}

In this section, we consider the first order advanced neutral difference equation

$$
\Delta[x(n)+p x(n+\tau)]-\sum_{i=1}^{r} q_{i}(n) x\left(n+\sigma_{i}\right)=0 ; \quad n \geq n_{0},
$$

where $\tau, r, \sigma_{1}, \sigma_{2}, \ldots \sigma_{r}$ are positive integers, $p$ is a real number and $\left\{q_{i}(n)\right\}$, $i=1,2, \ldots r$ are sequences of nonnegative real numbers defined on $N\left(n_{0}\right)$ and not identically zero for large values of $n$. We see that the equation (2) is a 
particular case of (1). We establish some sufficient conditions for the oscillation of all solutions of the difference equation (2).

In the sequel, unless otherwise specified, when we write a functional inequality, we assume that it holds for all sufficiently large $n$.

Theorem 1. Assume that

$$
\frac{\sigma^{\sigma}}{(\sigma-1)^{\sigma-1}} \sum_{i=1}^{r} \liminf _{n \rightarrow \infty} q_{i}(n)>1,
$$

where $p=0$, and $\sigma=\min _{1 \leq i \leq r} \sigma_{i}$. Then (2) is oscillatory.

Proof. We will prove that the advanced difference inequality

$$
\Delta x(n)-\sum_{i=1}^{r} q_{i}(n) x\left(n+\sigma_{i}\right) \geq 0 ; \quad n \geq n_{0}
$$

has no eventually positive solution. On the contrary, without loss of generality, assume that (4) has an eventually positive solution $\{x(n)\}$.

Setting $v(n)=\frac{x(n+1)}{x(n)}$ and dividing the inequality (4) by $x(n)$, we obtain

$$
\frac{1}{v(n)} \leq 1-\sum_{i=1}^{r} q_{i}(n) \prod_{l=1}^{\sigma_{i}-1} v(n+l) .
$$

Since $\{x(n)\}$ is nondecreasing, we have $v(n) \geq 1$.

From (3) and (5) we see that $\{v(n)\}$ is bounded above.

Let

$$
\liminf _{n \rightarrow \infty} v(n)=\beta
$$

we have

$$
\limsup _{n \rightarrow \infty} \frac{1}{v(n)}=\frac{1}{\beta} \leq 1-\sum_{i=1}^{r} \liminf _{n \rightarrow \infty} q_{i}(n) \beta^{\sigma_{i}-1}
$$

or

$$
\frac{1}{\beta} \leq 1-\sum_{i=1}^{r} \liminf _{n \rightarrow \infty} q_{i}(n) \beta^{\sigma_{i}-1} .
$$

Since $\beta^{\sigma_{i}} \geq \beta^{\sigma}$, for $i=1,2,3, \ldots, r$, we have

$$
\frac{1}{\beta} \leq 1-\sum_{i=1}^{r} \liminf _{n \rightarrow \infty} q_{i}(n) \beta^{\sigma-1}, \quad \text { for } \quad i=1,2, \ldots, r,
$$


or

$$
\sum_{i=1}^{r} \liminf _{n \rightarrow \infty} q_{i}(n) \leq \frac{\beta-1}{\beta^{\sigma}}
$$

But

$$
\frac{\beta-1}{\beta^{\sigma}} \leq \frac{(\sigma-1)^{\sigma-1}}{\sigma^{\sigma}}
$$

so

$$
\frac{\sigma^{\sigma}}{(\sigma-1)^{\sigma-1}} \sum_{i=1}^{r} \liminf q_{i}(n) \leq 1,
$$

which contradicts (3). Hence, (4) has no eventually positive solution.

Similarly, we can prove that the inequality

$$
\Delta x(n)-\sum_{i=1}^{r} q_{i}(n) x\left(n+\sigma_{i}\right) \leq 0, \quad n \geq n_{0}
$$

has no eventually negative solution.

The proof is complete.

Corollary 2. Assume that

$$
r\left[\prod_{i=1}^{r} \liminf _{n \rightarrow \infty} q_{i}(n)\right]^{\frac{1}{r}}>\frac{(\hat{\sigma}-1)^{\hat{\sigma}-1}}{\hat{\sigma}^{\hat{\sigma}}}
$$

where $p=0$, and $\hat{\sigma}=\frac{1}{r} \sum_{i=1}^{r} \sigma_{i}$. Then, (2) is oscillatory.

Proof. We will prove that (4) has no eventually positive solution. Assume, for the sake of contradiction, that (4) has an eventually positive solution $\{x(n)\}$. Applying the same procedure as we followed in the proof of Theorem 1, we have

$$
\frac{1}{\beta} \leq 1-\sum_{i=1}^{r} \liminf _{n \rightarrow \infty} q_{i}(n) \beta^{\sigma_{i}-1} .
$$

Using arithmetic and geometric mean inequality, we obtain

$$
\sum_{i=1}^{r} \liminf _{n \rightarrow \infty} \beta^{\sigma_{i}-1} \geq r\left(\prod_{i=1}^{r} \liminf _{n \rightarrow \infty} q_{i}(n) \beta^{\sigma_{i}-1}\right)^{\frac{1}{r}}
$$

which is the same as

$$
\sum_{i=1}^{r} \liminf _{n \rightarrow \infty} q_{i}(n) \beta^{\sigma_{i}-1} \geq r\left(\prod_{i=1}^{r} \liminf _{n \rightarrow \infty} q_{i}(n)\right)^{\frac{1}{r}} \beta^{\hat{\sigma}-1} .
$$


This implies

$$
\frac{1}{\beta} \leq 1-r\left(\prod_{i=1}^{r} \liminf _{n \rightarrow \infty} q_{i}(n)\right)^{\frac{1}{r}} \beta^{\hat{\sigma}-1},
$$

or

$$
r\left(\prod_{i=1}^{r} \liminf _{n \rightarrow \infty} q_{i}(n)\right)^{\frac{1}{r}} \leq \frac{\beta-1}{\beta^{\hat{\sigma}}}
$$

or

$$
r\left[\prod_{i=1}^{r} \liminf _{n \rightarrow \infty} q_{i}(n)\right]^{\frac{1}{r}} \leq \frac{(\hat{\sigma}-1)^{\hat{\sigma}-1}}{\hat{\sigma}^{\hat{\sigma}}}
$$

which contradicts (7). Hence the inequality (4) has no eventually positive solution.

Next, we consider the equation $(2)$ in case $p \neq 0$. In the sequel we need the following lemma.

Lemma 3. Let $\{x(n)\}$ be an eventually positive solution of (2). Set

$$
z(n)=x(n)+p x(n+\tau),
$$

we have

a) If $p<-1$, then $z(n)<0$ and $\Delta z(n)>0$ eventually.

b) If $-1<p<0$ and $\sum_{l=1}^{\infty}\left[\sum_{i=1}^{r} q_{i}(l)\right]=\infty$, then $z(n)>0$ and $\Delta z(n)>0$ eventually.

Proof. a) Since $q_{i}(n) \neq 0$, we have

$$
\Delta z(n)=\sum_{i=1}^{r} q_{i}(n) x\left(n+\sigma_{i}\right)>0 \quad \text { eventually }
$$

so $\{z(n)\}$ cannot be eventually identically zero. If $z(n)>0$ eventually, then

$$
z(n) \geq z(N)>0 \text { for all } n \geq N
$$

for sufficiently large $N \geq n_{0}$.

Since $p<-1$, we have

$$
z(n)=x(n)+p x(n+\tau)<x(n)-x(n+\tau)
$$

which implies that

$$
x(n+\tau)<x(n)-z(n) \leq x(n)-z(N) .
$$


Therefore,

$$
x(N+n \tau)<x(N)-n z(N) .
$$

Taking $n \rightarrow \infty$ in the above inequality, we have $x(n) \rightarrow-\infty$ as $n \rightarrow \infty$, which is a contradiction to $x(n)>0$.

b) We have

$$
\Delta z(n)=\sum_{i=1}^{r} q_{i}(n) x\left(n+\sigma_{i}\right)>0,
$$

for sufficiently large $n$. We shall prove that $z(n)>0$, eventually. Assume, for the sake of contradiction, that

$$
z(n)=x(n)+p x(n+\tau) \leq 0, \quad n \geq N
$$

That is,

$$
x(n) \leq-p x(n+\tau), \quad n \geq N,
$$

or

$$
x(n+\tau) \geq\left(\frac{-1}{p}\right) x(n) .
$$

Inductively,

$$
x(n+k \tau) \geq\left(\frac{-1}{p}\right)^{k} x(n) .
$$

On letting $k \rightarrow \infty$ in the above inequality, we get $x(n) \rightarrow \infty$ as $n \rightarrow \infty$. But

$$
\Delta z(n)=\sum_{i=1}^{r} q_{i}(n) x\left(n+\sigma_{i}\right) \geq M \sum_{i=1}^{r} q_{i}(n)
$$

for sufficiently large, where $M>0$. Summing (9) from $N$ to $n-1$, we obtain

$$
z(n)-z(N) \geq M \sum_{l=N}^{n-1}\left[\sum_{i=1}^{r} q_{i}(l)\right]
$$

which implies that $z(n) \rightarrow+\infty$ as $n \rightarrow \infty$.

This contradicts the hypothesis that eventually $z(n) \leq 0$.

Theorem 4. Suppose that

$$
\sum_{n=0}^{\infty} \sum_{i=1}^{r} q_{i}(n)=\infty
$$


and

$$
\frac{1}{1+p} \frac{\sigma^{\sigma}}{(\sigma-1)^{\sigma-1}} \sum_{i=1}^{r} \liminf _{n \rightarrow \infty} q_{i}(n)>1
$$

where

$$
-1<p<0, \quad \sigma=\min _{1 \leq i \leq r} \sigma_{i} \quad \text { and } \quad q_{i}(n+\tau) \leq q_{i}(n), \quad i=1,2, \ldots, r .
$$

Then, every solution of (2) is oscillatory.

Proof. On the contrary, let us assume that the equation (2) has an eventually positive solution $\{x(n)\}$. Set

$$
z(n)=x(n)+p x(n+\tau)
$$

and

$$
w(n)=z(n)+p z(n+\tau) .
$$

Then, by the case (b) of Lemma $3, z(n)>0, \Delta z(n)>0$.

Now, we shall prove that eventually $w(n)>0$. We have

$$
\begin{aligned}
\Delta w(n) & =\Delta z(n)+p \Delta z(n+\tau) \\
& =\sum_{i=1}^{r} q_{i}(n) x\left(n+\sigma_{i}\right)+p \sum_{i=1}^{r} q_{i}(n+\tau) x\left(n+\tau+\sigma_{i}\right) \\
& \geq \sum_{i=1}^{r} q_{i}(n) x\left(n+\sigma_{i}\right)+p \sum_{i=1}^{r} q_{i}(n) x\left(n+\tau+\sigma_{i}\right) \\
& =\sum_{i=1}^{r} q_{i}(n) z\left(n+\sigma_{i}\right) \geq 0 .
\end{aligned}
$$

Putting

$$
\lim _{n \rightarrow \infty} z(n)=\beta
$$

Then $\beta \leq \infty$.

$\operatorname{Case}(\mathbf{i})$ : If $\beta<\infty$, then

$$
\lim _{n \rightarrow \infty} w(n)=\beta(1+p)>0 .
$$

This implies $w(n)>0$. 
Case(ii) : Assume that $\beta=\infty$. Then there exist $M>0$ such that $z(n) \geq$ $M$, eventually. From this and (12), we have,

$$
\Delta w(n) \geq M \sum_{i=1}^{r} q_{i}(n) .
$$

Summing the above inequality from $n_{1}$ to $n-1$ and taking $n \rightarrow \infty$, we have

$$
w(n) \geq w\left(n_{1}\right)+M \sum_{n=n_{1}}^{\infty} \sum_{i=1}^{r} q_{i}(n) .
$$

By (10), we have $w(n) \rightarrow+\infty$ as $n \rightarrow \infty$. This shows that $w(n)>0$, eventually. Therefore, $w(n)>0$ for sufficiently large $n$. On the other hand,

$$
w(n)=z(n)+p z(n+\tau) \leq z(n)(1+p),
$$

or

$$
z\left(n+\sigma_{i}\right) \geq \frac{w\left(n+\sigma_{i}\right)}{1+p} .
$$

Using this in (12), we have,

$$
\Delta w(n)-\frac{1}{1+p} \sum_{i=1}^{r} q_{i}(n) w\left(n+\sigma_{i}\right) \geq 0 .
$$

By Theorem 1 and in view of condition (11) the inequality (13) has no eventually positive solution. This is a contradiction.

Lemma 5. Assume that $-1<p<0$ and $\tau>\sigma$, where $\sigma=\min _{1 \leq i \leq r} \sigma_{i}$. Then, the maximum value of $F(\beta)=\frac{\beta-1}{\beta^{\sigma}}\left(1+p \beta^{\tau}\right)$ on $[1, \infty)$ is $F\left(\beta^{*}\right)$, in which $\beta^{*} \in\left(1,(-p)^{\frac{-1}{\tau}}\right)$ is a unique real solution of the equation

$$
\beta\left(1+p \beta^{\tau}\right)+(\beta-1)\left[p \tau \beta^{\tau}-\sigma\left(1+p \beta^{\tau}\right)\right]=0 .
$$

Proof. The equation $F^{\prime}(\beta)=0$ is equivalent to

$$
\beta\left(1+p \beta^{\tau}\right)+(\beta-1)\left[p \tau \beta^{\tau}-\sigma\left(1+p \beta^{\tau}\right)\right]=0 .
$$

Put

$$
\phi(\beta)=\beta\left(1+p \beta^{\tau}\right)+(\beta-1)\left[\left(p \tau \beta^{\tau}-\sigma\left(1+p \beta^{\tau}\right)\right] .\right.
$$

We have,

$$
\phi(\beta)=1+p(\tau+1) \beta^{\tau}+p \beta^{\tau}(\tau-\sigma)-\sigma+(\beta-1) p \tau \beta^{\tau-1}(\tau-\sigma) .
$$


Since $\tau>\sigma \geq 1$, we have $\phi^{\prime}(\beta)<0$. On the other hand, we get

$$
\begin{gathered}
\phi(1)=1+p>0 \quad \text { and } \\
\lim _{\beta \rightarrow+\infty} \phi(\beta)=\lim _{\beta \rightarrow+\infty}\left[\beta\left(1+p \beta^{\tau}\right)+(\beta-1)\left(p \tau \beta^{\tau}-\sigma\left(1+p \beta^{\tau}\right)\right]=-\infty .\right.
\end{gathered}
$$

This implies that, $\phi$ is a decreasing function, starting from a positive value at $\beta=1$, and hence (14) has a unique real solution $\beta^{*} \in[1, \infty)$. Clearly, we see that $\beta^{*} \in\left(1,(-p)^{\frac{-1}{\tau}}\right)$ and $F(\beta) \geq 0$, for $\beta \in\left(1,(-p)^{\frac{-1}{\tau}}\right)$, which implies that $F\left(\beta^{*}\right)$ is the maximum value of $F(\beta)$ on $[1, \infty)$.

This completes the proof.

Theorem 6. Assume that $-1<p<0$ and (10) hold. Assume further that $\tau>\sigma$, where $\sigma=\min _{1 \leq i \leq r} \sigma_{i}, q_{i}(n+\tau) \geq q_{i}(n), i=1,2,3, \ldots, r$ for sufficiently large $n$ and

$$
\sum_{i=1}^{r} \liminf _{n \rightarrow \infty} q_{i}(n)>\frac{\beta^{*}-1}{\beta^{*^{\sigma}}}\left(1+p \beta^{\tau}\right),
$$

where $\beta^{*} \in[1, \infty)$ is defined as in Lemma 5. Then, every solution of (2) is oscillatory.

Proof. Assume the contrary. Without loss of generality, we may assume that the equation (2) has an eventually positive solution $\{x(n)\}$. Set

$$
z(n)=x(n)+p x(n+\tau)
$$

and

$$
w(n)=z(n)+p z(n+\tau) .
$$

By the case (b) of Lemma 3, we get $z(n)>0$ and $\Delta z(n)>0$ eventually. On the other hand,

$$
\Delta w(n)=\Delta(z(n)+p z(n+\tau)) \geq \sum_{i=1}^{r} q_{i}(n) z\left(n+\sigma_{i}\right) .
$$

Putting $\gamma(n)=\frac{z(n)}{z(n-1)}$, we get $\gamma(n) \geq 1$. Dividing $(16)$ by $z(n+1)$, we get

$$
\frac{1}{\gamma(n+1)} \leq 1+p\left[\frac{z(n+\tau+1)}{z(n+1)}-\frac{z(n+\tau)}{z(n+1)}\right]-\sum_{i=1}^{r} q_{i}(n) \frac{z\left(n+\sigma_{i}\right)}{z(n+1)}
$$

or

$$
\frac{1}{\gamma(n+1)} \leq 1+p[\gamma(n+2) \gamma(n+3) \ldots \gamma(n+\tau+1)
$$




$$
\begin{array}{r}
-\gamma(n+2) \gamma(n+3) \ldots \gamma(n+\tau+1)] \\
-\sum_{i=1}^{r} q_{i}(n) \gamma(n+2) \gamma(n+3) \ldots \gamma\left(n+\sigma_{i}\right) .
\end{array}
$$

Setting $\liminf \operatorname{in}_{n \rightarrow \infty} \gamma(n)=\beta$, we get $\beta \geq 1$. It is clear that $\beta$ is finite. From (17), we have

$$
\limsup _{n \rightarrow \infty} \frac{1}{\gamma(n+1)}=\frac{1}{\beta} \leq 1+p \beta^{\tau-1}(\beta-1)-\sum_{i=1}^{r} \liminf _{n \rightarrow \infty} q_{i}(n) \beta^{\sigma_{i}-1},
$$

or

$$
\sum_{i=1}^{r} \liminf _{n \rightarrow \infty} q_{i}(n) \beta^{\sigma_{i}-1} \leq 1+p \beta^{\tau-1}(\beta-1)-\frac{1}{\beta}
$$

or

$$
\sum_{i=1}^{r} \liminf _{n \rightarrow \infty} q_{i}(n) \leq \frac{(\beta-1)\left(1+p \beta^{\tau}\right)}{\beta^{\sigma}}=F(\beta)
$$

By Lemma 5, we have

$$
\sum_{i=1}^{r} \liminf _{n \rightarrow \infty} q_{i}(n) \leq F\left(\beta^{*}\right)=\frac{\left(\beta^{*}-1\right)\left(1+p \beta^{*^{\tau}}\right)}{\beta^{*^{\sigma}}}
$$

which contradicts condition (15). Hence, (2) has no eventually positive solution.

Theorem 7. Suppose that

$$
-\frac{1}{1+p} \frac{\left(\tau-\sigma^{*}+1\right)^{\tau-\sigma^{*}+1}}{\left(\tau-\sigma^{*}\right)^{\tau-\sigma^{*}}} \liminf _{n \rightarrow \infty} q_{i}(n)>1
$$

where $q_{i}(n) \leq q_{i}(n+\tau), i=1,2, \ldots, r$ for sufficiently large $n, p<-1, \sigma^{*}=$ $\max _{1 \leq i \leq r} \sigma_{i}, \tau>\sigma^{*}$. Then, every solution of (2) is oscillatory.

Proof. Assume the contrary. We may assume, with out loss of generality that $\{x(n)\}$ be an eventually positive solution of (2). Then by the case (a) of Lemma 3, we have $z(n)<0$ and $\Delta z(n)>0$ eventually. Setting

$$
z(n)=x(n)+p x(n+\tau)
$$

and

$$
w(n)=z(n)+p z(n+\tau)
$$


We can easily prove that $w(n)>0$ eventually. Now,

$$
\begin{gathered}
w(n)=z(n)+p z(n+\tau) \\
\leq z(n+\tau)(1+p),
\end{gathered}
$$

which implies that

$$
z\left(n+\sigma_{i}\right) \leq \frac{w\left(n+\sigma_{i}-\tau\right)}{1+p} .
$$

Therefore, it follows that

$$
\begin{aligned}
\Delta w(n) & =\Delta z(n)+p \Delta z(n+\tau) \\
& =\sum_{i=1}^{r} q_{i}(n) x\left(n+\sigma_{i}\right)+p \sum_{i=1}^{r} q_{i}(n+\tau) x\left(n+\tau+\sigma_{i}\right) \\
& \leq \sum_{i=1}^{r} q_{i}(n)\left[x\left(n+\sigma_{i}\right)+p x\left(n+\tau+\sigma_{i}\right)\right] \\
& =\sum_{i=1}^{r} q_{i}(n) z\left(n+\sigma_{i}\right) \\
& \leq \sum_{i=1}^{r} q_{i}(n) \frac{w\left(n+\sigma_{i}-\tau\right)}{1+p} .
\end{aligned}
$$

That is

$$
\Delta w(n)-\sum_{i=1}^{r} q_{i}(n) \frac{w\left(n+\sigma_{i}-\tau\right)}{1+p} \leq 0 .
$$

Setting

$$
\gamma(n)=\frac{w(n)}{w(n+1)}
$$

we get

$$
\gamma(n) \geq 1-\frac{1}{1+p} \sum_{i=1}^{r} q_{i}(n) \frac{w\left(n+\sigma_{i}-\tau\right)}{w(n+1)}
$$

or

$$
\gamma(n) \geq 1-\frac{1}{1+p} \sum_{i=1}^{r} q_{i}(n) \prod_{l=0}^{\tau-\sigma_{i}} \gamma(n-l)
$$

Putting

$$
\beta=\liminf _{n \rightarrow \infty} \gamma(n)
$$


we have $\beta \geq 1$. Now, (19) implies

$$
\beta \geq 1-\frac{1}{1+p} \sum_{i=1}^{r} \liminf _{n \rightarrow \infty} q_{i}(n) \beta^{\tau-\sigma_{i}+1} .
$$

Since

$$
\beta^{\tau-\sigma_{i}+1} \geq \beta^{\tau-\sigma^{*}+1}, i=1,2, \ldots, r
$$

we have,

$$
\beta \geq 1-\frac{1}{1+p} \sum_{i=1}^{r} \liminf _{n \rightarrow \infty} q_{i}(n) \beta^{\tau-\sigma^{*}+1},
$$

or

$$
\frac{-1}{1+p} \sum_{i=1}^{r} \liminf _{n \rightarrow \infty} q_{i}(n) \leq \frac{\beta-1}{\beta^{\tau-\sigma^{*}+1}} .
$$

But

$$
\frac{\beta-1}{\beta^{\tau-\sigma^{*}+1}} \leq \frac{\left(\tau-\sigma^{*}\right)^{\tau-\sigma^{*}}}{\left(\tau-\sigma^{*}+1\right)^{\tau-\sigma^{*}+1}}
$$

Then,

$$
\frac{-1}{1+p} \frac{\left(\tau-\sigma^{*}+1\right)^{\tau-\sigma^{*}+1}}{\left(\tau-\sigma^{*}\right)^{\tau-\sigma^{*}}} \sum_{i=1}^{r} \liminf _{n \rightarrow \infty} q_{i}(n) \leq 1,
$$

which contradicts condition (18). Hence, the equation (2) has no eventually positive solution. This completes the proof.

Theorem 8. Suppose that

$$
-\frac{1}{p} \frac{\left(\tau-\sigma^{*}+1\right)^{\tau-\sigma^{*}+1}}{\left(\tau-\sigma^{*}\right)^{\tau-\sigma^{*}}} \sum_{i=1}^{r} \liminf _{n \rightarrow \infty} q_{i}(n)>1,
$$

where $p<-1, \sigma^{*}=\max _{1 \leq i \leq r} \sigma_{i}, \tau>\sigma^{*}$. Then, every solution of (2) is oscillatory.

Proof. Assume the contrary. We may assume, without loss of generality, that the equation (2) has an eventually positive solution $\{x(n)\}$. Then by the case (a) of Lemma 3, we obtain $z(n)<0$ and $\Delta z(n)>0$ eventually. On the other hand, we have,

$$
z(n)>p x(n+\tau)
$$

or

$$
x(n+\tau)>\frac{1}{p} z(n),
$$


which implies that

$$
x\left(n+\sigma_{i}\right)>\frac{1}{p} z\left(n-\tau+\sigma_{i}\right) .
$$

Hence,

$$
\Delta z(n)=\sum_{i=1}^{r} q_{i}(n) x\left(n+\sigma_{i}\right)
$$

or

$$
\Delta z(n)>\frac{1}{p} \sum_{i=1}^{r} q_{i}(n) z\left(n-\tau+\sigma_{i}\right) .
$$

Setting $v_{n}=\frac{z(n)}{z(n+1)}$ and dividing $(22)$ by $z(n+1)$, we obtain

$$
v(n) \geq 1-\frac{1}{p} \sum_{i=1}^{r} q_{i}(n) \prod_{l=0}^{\tau-\sigma_{i}} \frac{z\left(n-\tau+\sigma_{i}+l\right)}{z\left(n-\tau+\sigma_{i}-1+l\right)}
$$

Taking lower limit on both sides of (23) and putting $\beta=\liminf _{n \rightarrow \infty} v(n)$, we have $\beta \geq 1$ and

$$
\begin{aligned}
& \beta \geq 1-\frac{1}{p} \sum_{i=1}^{r} \liminf _{n \rightarrow \infty} q_{i}(n) \beta^{\tau-\sigma_{i}+1} \\
& \geq 1-\frac{1}{p} \sum_{i=1}^{r} \liminf _{n \rightarrow \infty} q_{i}(n) \beta^{\tau-\sigma^{*}+1} .
\end{aligned}
$$

This implies that,

$$
-\frac{1}{p} \sum_{i=1}^{r} \liminf _{n \rightarrow \infty} q_{i}(n) \leq \frac{\beta-1}{\beta^{\tau-\sigma^{*}+1}}
$$

But

$$
\frac{\beta-1}{\beta^{\tau-\sigma^{*}+1}} \leq \frac{\left(\tau-\sigma^{*}\right)^{\tau-\sigma^{*}}}{\left(\tau-\sigma^{*}-1\right)^{\tau-\sigma^{*}-1}}
$$

using (25) in (24), we have

$$
-\frac{1}{p} \frac{\left(\tau-\sigma^{*}-1\right)^{\tau-\sigma^{*}-1}}{\left(\tau-\sigma^{*}\right)^{\tau-\sigma^{*}}} \sum_{i=1}^{r} \liminf _{n \rightarrow \infty} q_{i}(n) \leq 1,
$$

which contradicts the condition (21). Hence, the equation (2) has no eventually positive solution. This completes the proof. 


\subsection{Asymptotic Behavior}

In this section, we give some sufficient conditions for nonoscillatory solution of (1) tend to zero as $n \rightarrow \infty$. For this we need the following Lemma.

Lemma 9. Let $\{x(n)\}$ be a nonoscillatory solution of (1).

Put $z(n)=x(n)+p x(n+\tau)$.

(a) If $\{x(n)\}$ is eventually positive (negative), then $\{z(n)\}$ is eventually nondecreasing (nonincreasing).

(b) If $\{x(n)\}$ is an eventually positive (negative) and there exists a constant $\lambda$ such that

$$
p \leq \lambda<-1
$$

then eventually $z(n)<0(z(n)>0)$.

Proof. Let $\{x(n)\}$ be an eventually positive solution of (1). The case $\{x(n)\}$ is an eventually negative solution of (1) can be considered similarly.

(a) We have

$$
\Delta z(n)=\sum_{i=1}^{r} q_{i}(n) f\left(x\left(n+\sigma_{i}\right)\right) \geq 0,
$$

for all large $n$. Thus, $\{z(n)\}$ is an eventually nondecreasing.

(b) Assume the contrary. Since by $(\mathrm{a}),\{z(n)\}$ is a nondecreasing sequence, it follows that eventually either $z(n) \equiv 0$ or $z(n)>0$. Now $z(n) \equiv 0$ implies that

$$
\Delta z(n)=\sum_{i=1}^{r} q_{i}(n) f\left(x\left(n+\sigma_{i}\right)\right) \equiv 0,
$$

but this contradicts the fact that $q_{i}(n) \neq 0$ for infinitely many $n$. If $z(n)>0$, then

$$
x(n)>-p x(n+\tau) \geq-\lambda x(n+\tau) .
$$

Inductively,

$$
x(n+k \tau) \leq\left(\frac{-1}{\lambda}\right)^{k} x(n) .
$$

This shows that $x(n) \rightarrow 0$ as $n \rightarrow \infty$. Consequently, $z(n) \rightarrow 0$ as $n \rightarrow 0$.

This contradicts the fact that $\{z(n)\}$ is nondecreasing and $z(n)>0$ eventually.

This completes the proof. 
Theorem 10. Assume that (10) holds and there exists a constant $\mu$ such that

$$
p \leq \mu<-1 \text {. }
$$

Suppose further that if $|x| \geq k$ then $|f(x)| \geq k_{1}$, where $k$, and $k_{1}$ are positive constants. Then every nonoscillatory solution of (1) tends to 0 as $n \rightarrow \infty$.

Proof. With out loss of generality, we may suppose that $\{x(n)\}$ be an eventually positive solution of $(1)$, then $x(n)>0, x(n+\tau)>0$ and $x\left(n+\sigma_{i}\right)>0$ for sufficiently large values of $n$. Put

$$
z(n)=x(n)+p x(n+\tau)
$$

We first prove that $z(n) \rightarrow 0$ as $n \rightarrow \infty$. Note that (27) implies (26) with $\lambda$ replace by $\mu$. By Lemma 9 , we have $\{z(n)\}$ is eventually negative and nondecreasing. Therefore, $\lim _{n \rightarrow \infty} z(n)$ exists. Let

$$
\lim _{n \rightarrow \infty} z(n)=\beta
$$

Then $\beta \leq 0$. Now, suppose that $\beta<0$. Then by $(27)$, we have

$$
z(n)>p x(n+\tau)
$$

Thus,

$$
\beta \geq z(n)>p x(n+\tau)
$$

or

$$
\beta>p x(n+\tau)
$$

or

$$
x(n+\tau)>\frac{\beta}{p}>0 .
$$

Then by our hypothesis, there exists a positive constant $k_{1}$, such that

$$
f\left(x\left(n+\sigma_{i}\right)\right) \geq k_{1} .
$$

Now

$$
\begin{aligned}
\Delta z(n) & =\sum_{i=1}^{r} q_{i}(n) f\left(x\left(n+\sigma_{i}\right)\right) \\
& \geq k_{1} \sum_{i=1}^{r} q_{i}(n) .
\end{aligned}
$$


Summing the last inequality from $n_{1}$ to $n-1$, we obtain,

$$
z(n) \geq z\left(n_{1}\right)+k_{1} \sum_{s=n_{1}}^{n-1} \sum_{i=1}^{r} q_{i}(l) .
$$

Taking $n \rightarrow \infty$, we get $z(n) \rightarrow \infty$ as $n \rightarrow \infty$.

This is a contradiction to the fact that $\Delta z(n)>0$ and $z(n)<0$. So, we have

$$
\lim _{n \rightarrow \infty} z(n)=0
$$

Since $\lim _{n \rightarrow \infty} z(n)=0$, there exists a negative constant A such that $A \leq z(n)<$ 0 and so, by (27) we have

$$
x(n) \geq-\mu x(n+\tau)+A .
$$

Assume that $\{x(n)\}$ is not bounded. Then, there exists a subsequence $\left\{n_{k}\right\}$ of positive integers such that $x\left(n_{k}\right) \rightarrow \infty$ as $k \rightarrow \infty$ and

$$
x\left(n_{k}\right)=\max _{n_{0} \leq n \leq n_{k}} x(n), \quad k=1,2,3, \ldots
$$

From (28), we have

$$
x\left(n_{k}-\tau\right) \geq-\mu x\left(n_{k}\right)+A,
$$

or

$$
x\left(n_{k}\right) \geq-\mu x\left(n_{k}\right)+A,
$$

or

$$
(1+\mu) x\left(n_{k}\right) \geq A,
$$

which as $k \rightarrow \infty$ leads to a contradiction.

Now suppose that

$$
\limsup _{n \rightarrow \infty} x(n)=\delta>0 .
$$

Then, there exists a subsequence $\left\{n_{k}\right\}$ of positive integers such that $x\left(n_{k}\right) \rightarrow \delta$ as $k \rightarrow \infty$ and

$$
x\left(n_{k}\right)=\max _{n_{0} \leq n \leq n_{k}} x(n) .
$$

Then, from (27), we have

$$
z(n)=x(n)+p x(n+\tau) \leq x(n)+\mu x(n+\tau),
$$

or

$$
z(n-\tau) \leq x(n-\tau)+\mu x(n),
$$


or

$$
z\left(n_{k}-\tau\right) \leq x\left(n_{k}-\tau\right)+\mu x\left(n_{k}\right)
$$

or

$$
x\left(n_{k}-\tau\right) \geq z\left(n_{k}-\tau\right)-\mu x\left(n_{k}\right)
$$

or

$$
\delta \geq \lim _{k \rightarrow \infty} x\left(n_{k}-\tau\right) \geq-\mu \delta .
$$

Since $-\mu>1$ and $\delta>0$, it follows that $\alpha=0$, that is, $x(n) \rightarrow 0$ as $n \rightarrow \infty$. The arguments when $\{x(n)\}$ is an eventually negative solution of (1) is similar.

Theorem 11. Suppose there exists positive constants $M, q_{i}, i=1,2,3, \ldots, r$ such that

$$
\begin{gathered}
q_{i}(n) \geq q_{i}, \quad i=1,2,3, \ldots, r, \quad n \geq n_{0}, \\
|f(x)| \geq M|x|, \forall, x \in R
\end{gathered}
$$

and

$$
p<-1
$$

Then, every solution of (1) tends to zero as $n \rightarrow \infty$.

Proof. Without loss of generality, we may assume that $\{x(n)\}$ be an eventually positive solution of $(1)$. Then by Lemma $9,\{z(n)\}$ is eventually negative and nondecreasing. So $\lim _{n \rightarrow \infty} z(n)$ exists. Put

$$
\lim _{n \rightarrow \infty} z(n)=\beta
$$

Summing the equation (1) from $n$ to $\infty$ for $n \geq n_{0}$, we obtain

$$
-z(n)=-\beta+\sum_{l=n}^{\infty} \sum_{i=1}^{r} q_{i}(n) f\left(x\left(l+\sigma_{i}\right)\right), \quad n \geq n_{0} .
$$

Now by (29) and (30), we have

$$
M\left(\sum_{i=1}^{r} q_{i}\right) \sum_{l=n}^{\infty} x\left(l+\sigma_{i}\right) \leq \beta-z(n)<\infty,
$$

which implies that $x(n) \rightarrow 0$ as $n \rightarrow \infty$. The proof is similar when $\{x(n)\}$ is eventually negative. 
A. Murugesan, K. Shanmugavalli

\section{References}

[1] R. P. Agarwal, Difference Equations and Inequalities: Theory, Methods, and Applications, Marcd Dekkr, New York, 1999.

[2] R.P. Agarwal and P. J. Y. Wong, Advanced Topics in Difference Equations, Kluwer, Dodrecht, 1997.

[3] G. E. Chatzarakis and I. P. Stavroulakis, Oscillations of advanced difference equations with variable arguments, Electron. J. Qual. Theory Diff. Equ., 79 (2012), 1-16, doi: 10.14232/ejqtde.2012.1.79.

[4] M. P. Chen, B. S. Lalli and J. S. Yu, Oscillation in neutral delay difference equations with variable coefficients, Comput. Math. Appl., 29 (1995), 5-11.

[5] I. Györi and G. Ladas, Oscillation Theory of Delay Differential Equations with Applications, Oxford University Press, Oxford, 1991.

[6] D. C. Huong, Oscillation and convergence for a neutral difference equation, VNU Journal of Science, Mathematics-Physics, 24 (2008), 133-143.

[7] A. Murugesan, Oscillation of neutral advanced difference equation, Glob. J. Pure. Appl. Math., 9 (2013), 83-92.

[8] X. H. Tang and X. Lin, Necessary and sufficient conditions for oscillation of first order nonlinear neutral difference equations, Comput. Math. Appl., 55 (2008), 1279-1292.

[9] X. H. Tang, Asymptotic behavior of solutions for neutral difference equations, Comput. Math. Appl., 44 (2002), 301-315.

[10] E. Thandapani, R. Arul and P. S. Raja, Oscillation of first order neutral delay difference equations, Appl. Math. E-Notes, 3 (2003), 88-94. 